\title{
A Large Number of Herbig-Haro Candidates Found by the Contrast Enhancement and Unsharp Masking Technique
}

\author{
Katsuo Ogura \\ Kokugakuin University, Higashi, Shibuya-ku, Tokyo 150, Japan
}

\begin{abstract}
About 1300, 60 and 70 Herbig-Haro candidates have been picked up in the Ori A/L1641, Ori B/L1630 and NGC 2264/Mon OBl regions, respectively, on deep Schmidt plates. The contrast enhancement and unsharp masking technique has been applied simultaneously. Unsharp masking applied to an objective-prism Schmidt plate for the Carina Nebula region has yielded the detection of $38 \mathrm{H} \alpha$ emission stars, of which 13 are new findings.
\end{abstract}

\section{Introduction}

Herbig-Haro (HH) objects are small nebulae (size 1 arcmin) found in star forming dark clouds. They are shock-excited by hypersonic, often well-collimated winds emanating from YSOs (see, for a recent review, Ray \& Mundt 1993). They are quite interesting as a source of information not only on mass ejection in the earliest stage of stellar evolution but also on the phenomena of astrophysical jets in general.

The simplest method of searching for $\mathrm{HH}$ objects is to take objective-prism Schmidt observations in the red to detect small emission nebulae. However, since this method cannot reach very deep, it has seldom been used in recent years. The more standard search method is wide-band imaging to pick up candidate objects, followed by low-dispersion slit spectroscopy to confirm their nature. Recent popularization of CCD cameras has opened the second way to the followup observations, i.e. narrow-band imaging on and off the strong emission lines (usually [SII] 6717/6731) of $\mathrm{HH}$ objects. These show up only on the on-line images and disappear on the off-line images ("continuum frames"). The number of hitherto known $\mathrm{HH}$ objects is about 200.

As with many other sorts of celestial objects, more $\mathrm{HH}$ objects are found as we go fainter. So our intention is to make a deeper and more extensive search for $\mathrm{HH}$ candidates than has so far been attempted. We utilized the wide field of view of Schmidt plates and combined the modern photographic technique of contrast enhancement and unsharp masking (Malin 1977, 1981, 1994). The target fields are Ori A/Lynds 1641, Ori B/Lynds 1630 and NGC 2264/Mon OB1, all of which are well-known star forming regions accessible from both the northern and southern hemispheres. The result is the detection of a large number of $\mathrm{HH}$ candidates, especially in Ori A/L1641. 


\section{Observations and Plate Inspection}

Deep direct plates in the red were obtained with the UK Schmidt for the above three regions. For NGC 2264 we also used a red plate of a larger scale taken at the prime focus of the $3.9 \mathrm{~m}$ Anglo Australian Telescope (AAT). These plates were contrast-enhanced on films with the unsharp masking technique applied simultaneously in various degrees depending on the darkness of the plate background. The copy films were scanned with a binocular microscope to pick up small nebulae which look like $\mathrm{HH}$ objects, e.g. consisting of multiple knots. This photographic technique is extremely powerful. It is found that the dark clouds are in fact not completely dark but rich in faint nebulosities of various size and morphology, in particular having almost innumerable tiny nebulae. We have rejected nebulae which look more or less symmetric, since they may be galaxies. Also rejected are those which are very diffuse and/or associated with visible stars, since these are properties of reflection nebulae.

Table 1. Number of known and candidate HH Objects in three Fields

\begin{tabular}{lrrr}
\hline Region & L1641 & L1630 & NGC 2264/ \\
& & & Mon OB1 \\
\hline No. of known HHs & 39 & 24 & 4 \\
No. of candidate HHs & 1292 & 60 & 73 \\
\hline \hline
\end{tabular}

\section{Results}

The numbers of the objects picked up in the three fields are listed in Table 1. These fields include the less dense, outer regions of the dark clouds. Plate inspection is only preliminary in L1630. Really surprising is the large number of the L1641 objects. We suspect that at least 25 per cent of the objects in L1641 and about 40 per cent of the L1630 and NGC 2264/Mon OB1 objects are bona fide $\mathrm{HH}$ objects based on two reasons. First, many of them are elongated or composed of several knots aligned with common orientations, with position angles $\sim 150^{\circ}$ in L1641 and L1630 and $\sim 45^{\circ}$ in NGC 2264, a phenomenon found in known $\mathrm{HH}$ objects in L1641 (Reipurth 1989, Ogura and Walsh 1991). Second, we have made multi-object fibre spectroscopy for some 320 objects in L1641, confirming the $\mathrm{HH}$ nature for about $90 \mathrm{objects;} \mathrm{roughly} 70$ objects were too faint to classify (Ogura et al. 1994).

What then is the nature of the rest of the objects? We suspect that there are practically no supernova remnants and planetary nebulae among our objects, since the survey fields are of high Galactic latitude except for NGC 2264/Mon $\mathrm{OB} 1$ and in or around dense dark clouds or HII regions. As mentioned above, particular attention has been paid to avoid the inclusion of reflection nebulae. However, since they are most abundantly found in star-forming regions and more or less resemble $\mathrm{HH}$ objects, we suspect that they are the major source of contamination in our lists. The second possibility among the L1641 and L1630 objects is galaxies, since the outer Orion region, especially to the southwest of 
L1641, is rich in distant galaxies. As a third possibility some of our objects could be multiple stars. If the focus is locally poor in making the contrast-enhanced and unsharp-masked copies, they could easily be confused as HH candidates, because our objects are mostly small (10-30 arcsec) in size compared to known HH objects.

\section{Astrophysical Significance}

This work serves as a starting point for various follow-up observations, such as low- and high-dispersion spectroscopy and IR imaging, which will then provide a great deal of statistical information on $\mathrm{HH}$ objects and their exciting sources. However, we can make some arguments of astrophysical interest based on the present data. Since the exciting sources of $\mathrm{HH}$ objects are Young Stellar Objects (YSOs) embedded in dense clouds, they can be detected as IRAS point sources (Cohen and Schwartz 1987; Cohen 1990). However, only a small number of our objects are found located close to and can therefore be associated with IRAS sources: 47 objects with 38 IRAS sources in L1641, 19 with 15 in L1630 and 22 with 14 in NGC 2264/Mon OB1. In L1641 the lowest mass of the YSOs detected by IRAS is a little bit over $1 \mathrm{M}_{\odot}$. This means that there must be a large number $(\sim 300)$ of active YSOs of very low mass (less than $\left.1 \mathrm{M}_{\odot}\right)$ in L1641.

Also we can make a guess on the duration of the period for which each star shows $\mathrm{HH}$ activity in its early stages of evolution. Let $\mathrm{N}$ and $\mathrm{n}$ be the total numbers of $\mathrm{HH}$ objects and of pre-main sequence stars in a star-forming region, respectively. Their ratio should be related to that of the duration of $\mathrm{HH}$ activity $(\tau)$ and the entire lifetime of pre-main sequence evolution $(t)$ :

$$
\tau / \mathrm{t}=\mathrm{N} / \mathrm{n}
$$

In $L 1641$ we have $\mathrm{N} \sim 300$, as seen above, and $\mathrm{n} \sim 20000$. The latter is derived from the $\mathrm{J}, \mathrm{H}$ and $\mathrm{K}$ band imaging observations made by Strom et al. (1993) who detected about 1800 stars in 0.77 square degrees in L1641. Here some allowance has been made for the fact that our survey area includes the outer regions of L1641 as well, where the number density of embedded stars should be less than that of its central part. Then we have $\tau / \mathrm{t} \sim 0.015$; i.e., $\mathrm{HH}$ activity takes place during (probably the first) 1.5 per cent of the pre-main sequence lifetime. If we adopt $\mathrm{t} \sim 2 \times 10^{7} \mathrm{yr}$ for low mass stars, we get $\tau \sim 3 \times 10^{5} \mathrm{yr}$. This is roughly in agreement with the conclusion obtained by Mundt et al. (1987) who used a far smaller sample of $\mathrm{HH}$ objects in the Taurus dark cloud.

\section{Objects of Special Interest}

Among our $\mathrm{HH}$ candidates there are some groups of objects which attract special attention. First, several dozens of the L1641 objects show a fine, jet-like morphology, again sharing approximately the same orientation of position angle $\sim 150^{\circ}$. They are similar to known HH jets typified by the HH 34 jet (Reipurth \& Heathcote 1992), but at the same time differ in the lack of bow shocks ahead of the jets. Second, in the NGC 2264 region there is a concentration of boat-like 
structures, once again aligning with a similar orientation of position angle $\sim 45^{\circ}$. We suspect that they represent the cavities excavated by the outflow activities. Third, two pairs of giant (linear size $\sim 1$ pc) bow shock structures have been found associated with known $\mathrm{HH}$ jets. Located symmetrically on both sides of HH $1 / 2$ in L1641 and of HH 124 in NGC 2264, they have been designated as HH 1/2-NW, HH 1/2-SE, HH 124-W and HH 124-E. The HH nature of all of them has been confirmed by narrow band imaging on and off [SII] $6717 / 6731$ using the Kiso Schmidt in conjunction with a CCD camera. For HH 1/2-NW, low dispersion spectroscopy has also been obtained with the AAT. This object is so prominent that everyone who looked at the POSS print must have noticed it, but practically no paper has so far been writen on it. This is probably due to the difficulty of its interpretation, to which, however, the presence of $\mathrm{HH}$ $1 / 2$-SE of very similar morphology provides the key. Multiple bow shocks are known in a few HH objects such as HH 34 (Reipurth and Heathcote 1992) and are interpreted as evidence for the episodic nature of the outflow activity of the $\mathrm{HH}$ exciting sources. The giant bow shocks associated with $\mathrm{HH} 1 / 2$ and $\mathrm{HH} 124$ provide further, beautiful examples of this phenomenon and, with the dynamical age of $2 \times 10^{4} \mathrm{yr}$ in the both pairs, extend its timescale by nearly an order of magnitude.

\section{Search for Emission-Line Stars in the Carina Nebula Region}

The effect of the unsharp-masking technique can be shown more clearly in detecting faint objects in regions where the nebular background is very strong. The Carina Nebula is the brightest HII region in the southern sky and is associated with several young open clustes $\operatorname{Tr} 14, \operatorname{Tr} 15, \operatorname{Tr} 16, \mathrm{Cr} 228$ and $\operatorname{Cr} 232$. Searches for emission-line stars in this region have been attempted by several authors (e.g. Schwartz et al. 1990) and have shown the presence of a sizable number of $\mathrm{H} \alpha$ emission stars, many of which are Be stars. However, the extreme brightness of the nebular background hampers the detection of faint objects. This problem can be by-passed by applying the unsharp masking technique, since the dynamic range of photographic plates is very wide and so objective-prism plates still keep information on stellar spectra unless they are saturated.

We obtained a deep objective-prism plate in the red for the Carina Nebula region with the Curtis Schmidt at Cerro Tololo Inter-American Observatory and searched for $\mathrm{H} \alpha$ emission stars on the unsharp-masked copies. As a result, 38 objects, of which 13 are new findings, have been detected. Some, although not very many, of them are found to be located in the region where the nebula is very bright, showing the effect of unsharp masking.

As for the nature of these emission-line stars we do not have much information at the moment and need follow-up observations. But they cannot be $T$ Tauri stars associated with the Carina Nebula, since, with the distance of 2.7 kpc (Harvey et al. 1979) or $3.2 \mathrm{kpc}$ (Massey and Johnson 1993), their absolute magnitudes of $\mathrm{M}_{r} \lesssim+2.5 \mathrm{mag}$ would be too bright for such identification. Their spatial distribution is similar to that of known Be stars in this region. So we suspect that they are more likely to be Be or Ae stars. 


\section{Acknowledgements}

This work has been done in collaboration with D. F. Malin of the AAO, J. R. Walsh of ST-ECF, ESO and M. Shinohara of Komazawa University, Tokyo.

\section{References}

Cohen M., 1990, ApJ, 354, 701

Cohen M. \& Schwartz R.D., 1987, ApJ, 316, 311

Harvey P.M., Hoffmann W. F. \& Campbell M. F., 1979, ApJ, 227, 114

Malin D.F., 1977, AAS Photo Bulletin, 16, 10

Malin D.F., 1981, AAS Photo Bulletin, 27, 4

Malin D.F., 1994, these proceedings

Massey P. \& Johnson J., 1993, AJ, 105, 980

Mundt R., Brugel E.W. \& Buhrke, T., 1987, ApJ, 319, 275

Ogura K., Noumaru J., Walsh J. R. \& Malin D. F., 1994, in preparation

Ogura K. \& Walsh J. R., 1991, AJ, 101, 185

Ray T. \& Mundt R., 1993, in Proc. STScI Symp. on Astrophysical Jets, M. Fall, C. O'Dea, M. Livio \& D. Burgarella, eds, (Cambridge University Press), p. 145

Reipurth B., 1989, A\&A, 220, 249

Reipurth B. \& Heathcote S., 1992, A\&A, 257, 693

Schwartz R.D., Persson S.E. \& Hammann F. W., 1990, AJ, 100, 793

Strom K. M., Newton G., Strom S.E., Seaman R.l., Carrasco L., Cruz-Gonzalez L., Serrano A. \& Grasdalen G. L., 1989, ApJS, 71, 183

Strom K. M., Strom S. E., Wolff S.C., Morgan J. \& Wenz M. 1986, ApJS, 62, 39

\section{Discussion}

Briceno: What is the reason to believe that the common elongation of $\mathrm{HH}$ candidates along approximately the same position angle in the same dark cloud is a criterion for them to be actually $\mathrm{HH}$ objects?

Ogura: The common direction of elongation or knot alignment is not necessarily a criterion of HH objects. But many of the known HH objects in L1641 share this empirical tendency (Reipurth 1989, Ogura \& Walsh 1991).

Briceno: What is the limiting magnitude of your objective prism plate in the Carina Nebula region?

Ogura: The limiting magnitude is about $15.5 \mathrm{mag}$ in the $\mathrm{R}$ band.

Hidayat: What is the astrophysical meaning of the knot alignment in view of the Galactic structure? 
Ogura: The orientation of elongation or knot alignment is close to the overall magnetic field vector in L1641 (Strom et al. 1986, Reipurth 1989). This can be explained in the following way. When a cloud fragment contracts it should occur preferentially along the local magnetic field, eventually forming a system of a protostar and its circumstellar disk, which is flattened perpendicular to the magnetic field. The outflow from the protostar should be channeled toward its polar direction due to some magnetic effect or to the least resistance.

Hidayat: What are the linear distances of the giant bow shocks from the exciting sources? Do they conform with the theory of mass ejection?

Ogura: In both HH $1 / 2$ and HH 124 the giant bow shocks are some $3 \mathrm{pc}$ away from their exciting sources. The density of the ambient gas is relatively low in the inter-cloud core regions and so the outflows can reach this distance before exhausting their kinetic energy.

MacConnell: Do you see any continuum on the objective-prism plate for the $\mathrm{H} \alpha$ emission objects? If so, what do they look like? Are there any molecular bands?

Ogura: Yes. The continua are almost flat and do not show molecular bands in most of our $\mathrm{H} \alpha$ emission stars in the Carina Nebula region. 